\title{
BMJ Open Comparing rates and characteristics of emergency department presentations related to pharmaceutical opioid poisoning in Australia: a study protocol for a retrospective observational study
}

\author{
Tina Lam, ${ }^{1}$ Jane Hayman, ${ }^{1,2}$ Janneke Berecki-Gisolf, ${ }^{1,2}$ Paul Sanfilippo, ${ }^{1,3}$ \\ Dan I Lubman, ${ }^{1,3}$ Suzanne Nielsen (D) 1,3
}

To cite: Lam T, Hayman J, Berecki-Gisolf J, et al. Comparing rates and characteristics of emergency department presentations related to pharmaceutical opioid poisoning in Australia: a study protocol for a retrospective observational study. BMJ Open 2020;10:e038979. doi:10.1136/ bmjopen-2020-038979

- Prepublication history for this paper is available online. To view these files, please visit the journal online (http://dx.doi org/10.1136/bmjopen-2020038979).

Received 31 March 2020 Revised 16 July 2020 Accepted 21 August 2020

D) Check for updates

(c) Author(s) (or their employer(s)) 2020. Re-use permitted under CC BY-NC. No commercial re-use. See rights and permissions. Published by BMJ.

For numbered affiliations see end of article.

\section{Correspondence to} Associate Professor Suzanne Nielsen;

suzanne.nielsen@monash.edu

\section{ABSTRACT}

Introduction and aims Pharmaceutical opioids are an important contributor to the global 'opioid crisis', and are implicated in $70 \%$ of Australia's opioid-related mortality. However, there have been few studies which consider the relative contribution of different pharmaceutical opioids to harm.

We aim to compare commonly used pharmaceutical opioids in terms of (1) rates of harm, and (2) demographic and clinical characteristics associated with that harm.

Method and analysis Observational study of emergency department presentations for non-fatal poisoning related to pharmaceutical opioid use. Data from 2009 to 2019 will be extracted from the Victorian Emergency Minimum Dataset which contains data from public hospitals with dedicated emergency departments in Victoria, Australia's second most populous state. A combination of free-text and International Classification of Diseases 10th Revision codes will be used to identify relevant cases, with manual screening of each case to confirm relevance. We will calculate supply-adjusted rates of presentations using Poisson regression for all pharmaceutical opioid cases identified, separately for nine commonly prescribed pharmaceutical opioids (buprenorphine, codeine, fentanyl, methadone, morphine, oxycodone, oxycodone-naloxone, tapentadol, tramadol), and for a multiple opioid category. We will use multinomial logistic regression to compare demographic and clinical characteristics, such as triage category, across opioid types.

Ethics and dissemination This work is conducted under approval 21427 from the Monash University Human Research Ethics Committee for ongoing injury surveillance. As per conditions of approval, cells of $<5$ will not be reported, though zeroes will be preserved. We will present project findings in a peer-reviewed journal article as well as at relevant scientific conferences.

\section{INTRODUCTION}

Over the past two decades, opioid-related deaths have rapidly escalated in high-income regions, and despite a range of preventative strategies being trialled, the situation
Strengths and limitations of this study

- Indicator of population-level opioid harm.

- Cases have been selected using International Classification of Diseases 10th Revision (ICD) codes and free-text searches, increasing the capture of relevant cases over studies that use ICD codes alone.

- Manual confirmation of individual case eligibility, as opposed to assuming eligibility via ICD code or keyword alone.

- Inclusion of nine different types of pharmaceutical opioids.

- Around two-thirds of opioid-related cases have a specific opioid recorded, enabling inclusion in our analysis. One-third do not. This means that the rates of poisoning are almost certainly an underestimation, though we have no reason to believe that this would create a bias in comparisons between individual opioids.

continues to worsen. ${ }^{1}$ Every day in Australia, there are almost 150 hospitalisations for opioid harms, ${ }^{2}$ and there was a $30 \%$ increase in US Emergency Department (ED) visits for opioid overdoses from 2016 to 2017. ${ }^{3}$ Given substantial harms are attributed to pharmaceutical opioids (eg, $70 \%$ of opioid-related mortality in Australia), ${ }^{4}$ this has highlighted how important it is to understand the risk profiles associated with individual opioids.

Recent US research has revealed the risk profile of some pharmaceutical opioids may vary by potency. ${ }^{5}$ Similar research using Australian ambulance attendance data found that different pharmaceutical opioids have distinct patterns of harm. ${ }^{6}$ For example, the highest rates of opioid-related ambulance attendance were accounted for by the lowest potency opioid, different opioids were associated with accidental overdoses versus suicidal 
intent, and the availability and formulation of the opioid appeared important in explaining patterns of harm. ${ }^{6}$

There is a need for further research to extend these findings with other datasets, and particularly to focus on the outcome of opioid poisoning. Opioid poisoning is also referred to as 'opioid overdose', and is an acute condition resulting from the absorption of excessive amounts of opioids into the body. ${ }^{7}$ Non-fatal overdose is associated with increased risk of later fatal overdose, and is estimated to occur 13-30 times more frequently than fatal overdose. ${ }^{89}$

ED data are routinely used to monitor for trends in opioid poisoning. ${ }^{3}$ Although some studies have disaggregated results by heroin and pharmaceutical opioids, ${ }^{10}$ few ED studies have differentiated between the individual pharmaceutical opioids involved. Relative harms with pharmaceutical opioids have been examined through coded ambulance attendances ${ }^{6}$; however, there are many patients who self-present to the ED without the use of an ambulance service, and these patients may represent a unique population.

This paper outlines a study to better understand pharmaceutical opioid poisonings treated within EDs to inform clinical treatment and prevention strategies.

We write this protocol to maximise transparency ${ }^{11} 12$ as the study is supported by an untied educational grant from Seqirus, who are the Australian distributors of Palexia (tapentadol) and Tramal (tramadol). Tapentadol was introduced to the Australian market in 2011, subsidised in 2013 and is currently the fourth most commonly prescribed opioid in Victoria. ${ }^{6}$

\section{METHODS}

\section{Study aims}

The proposed study has two key objectives:

1. To compare the supply-adjusted rates between 2009 and 2019 for Victorian ED opioid poisoning presentations across nine common pharmaceutical opioids and one 'multiple opioid' category.

2. To examine demographics and other presentation characteristics including severity and context for opioid poisoning presentations with different pharmaceutical opioids.

We will answer the following research questions:

1. Do the supply-adjusted rates of ED presentations differ over time and across pharmaceutical opioids?

2. Does the severity of presentation (as measured by triage category) or other characteristics vary by opioid type?

\section{Study design}

The study design is a retrospective observational study that uses administrative ED data. Existing pharmaceutical opioid poisoning-related ED presentations are identified, coded and supply-adjusted rates are calculated to compare harms across opioids commonly prescribed within Australia including fentanyl, buprenorphine, oxycodone, oxycodone-naloxone, codeine, morphine, methadone, tramadol and tapentadol.

\section{Study population}

Data come from public ED presentations in Victoria, Australia's second most populous jurisdiction, representing $26 \%$ of the Australian population. ${ }^{13}$ Victoria's per capita rate for unintentional pharmaceutical-opioid induced deaths is comparable with other Australian jurisdictions ${ }^{14}$ and previous work demonstrated that Victorian rates of ambulance attendance for extramedical pharmaceutical opioid use were broadly consistent with other jurisdictions. ${ }^{6}$ Australia has the eighth highest per capita licit pharmaceutical opioid consumption in the world. ${ }^{2}$

This population-wide study has a catchment area of the entire Victorian state, for the past 10 years (July 2009June 2019), for individuals aged 12 years of age and older. Data will be obtained from all 38 public hospitals with a 24-hour ED contributing to the Victorian Emergency Minimum Dataset (VEMD).

\section{ED data}

Australia has a universal healthcare scheme which covers the cost of public hospital services. ${ }^{15}$ It is mandatory for all public ED presentations in the state of Victoria to be entered into the VEMD by ED staff. ${ }^{16}$ The database is managed by the state government's Department of Health and Human Services. ${ }^{16}$

The VEMD ${ }^{16}$ captures ED presentations related to drug poisoning and overdose, an important populationlevel indicator of opioid-related harm. Using approaches consistent with previous work with ambulance datasets, ${ }^{17}$ these ED data provide a useful way to identify if harms are emerging with specific pharmaceutical drugs, and to understand the nature of these harms. These data can inform both the relative frequency and severity of presentations associated with different pharmaceutical opioids. These findings will complement previous work, ${ }^{17}$ and when these reports are considered together, will advance our knowledge on the prevalence and severity of opioidrelated harms involving different pharmaceutical opioids.

The VEMD manual ${ }^{16}$ documents the standardised procedures used to ensure the accuracy, validity, completeness and coherence of captured and reported data across datasets and over time. These procedures include that data submitted by the health services are checked for valid values and compliance with VEMD business rules, and a requirement for corrections and resubmissions until the service has a 'clean' (zero rejection) submission. The Department of Health and Human Services circulates a monthly compliance report to monitor the completeness of submissions to the VEMD, runs regular analyses and sends out compliance emails when reporting deadlines are missed. The VEMD is also subject to audits by the Health Data Integrity Unit in the Victorian Agency for Health Information.

In addition to data quality systems with the VEMD database, an experienced data analyst will manually check all 
extracted cases to ensure there are no inconsistencies between coded cases and the narrative (eg, excluding cases where only non-opioid drugs are stated in the narrative, or the pharmaceutical opioid menioned was not implicated in a poisoning event) to confirm the ED presentation relates to a pharmaceutical opioid overdose or poisoning.

\section{Opioids of interest}

We will extract all VEMD records pertaining to pharmaceutical opioid poisoning, and concentrate our analyses on the subset of data where the specific pharmaceutical opioid(s) involved in the presentation is documented.

We will analyse the data by 9 specific opioids, with a 10 th category for presentations involving multiple opioids. The opioid categories we will analyse are: (1) buprenorphine, (2) codeine, (3) fentanyl, (4) methadone, (5) morphine, (6) oxycodone, (7) oxycodone-naloxone, (8) tapentadol, (9) tramadol, and (10) multiple opioids.

The first nine categories represent the opioids most commonly used for analgesia in outpatient settings in Australia. Our previous work demonstrated that less common drugs such as pethidine and dextropropoxyphene are captured in too few numbers to report on, given the requirement to suppress cell sizes of less than five. ${ }^{6}$ We will not examine opioids that are rarely used (eg, dextropropoxyphene), not available in Australia (such as hydrocodone), and those used only in inpatient settings (such as alfentanil and sufentanil).

Based on previous research with ambulance attendances, we expect that records involving multiple pharmaceutical opioids will represent less than $10 \%$ of cases. ${ }^{6}$ Given the small expected numbers, the inclusion of the 'multiple opioids' group is not expected to affect conclusions drawn from the study. We will quantify the individual opioids within the multiple opioid category, but not attempt to attribute outcomes to individual opioids within the multiple opioid category.

\section{Case identification and coding}

Records of opioid-poisoning cases from July 2009 to June 2019 will be extracted from the VEMD by the Victorian Injury Surveillance Unit (VISU) at Monash University in April/May 2020 following external protocol peer review.

Inclusion of a case is based on the documentation that the opioid was involved in the ED presentation, and this is established based on the (1) narrative data recorded in the VEMD, and/or (2) and International Classification of Diseases 10th Revision, Australian modification (ICD10-AM) poisoning diagnosis codes T40.2, T40.3, T40.4 (see table 1 for an overview).

\section{Selection of narrative cases}

A text search will be conducted of the 'Description of Event' variable, which contains a text narrative describing the opioid type and circumstances of the injury (presence of overdose or poisoning). The first part of the text search will be for drug names and brand names, and cases will be coded into individual opioid types (eg, codeine, methadone). Next, individual opioid types will be further coded as a poisoning or overdose if (1) the following terms are listed in the 'Description of Event' text variable: 'overdose' 'od' 'o/d' 'poisoning' 'drug abuse' 'toxicity' 'poison' 'self harm' 'suicide' OR (2) there is an ICD10-AM T40 'poisoning by narcotics and psychodysleptics' code anywhere within the three VEMD diagnosis codes.

\section{Selection of ICD10-AM coded cases}

Where the 'Description of Injury Event' variable does not contain a drug name (ie, not selected via the narrative search described above), cases of pharmaceutical opioid poisoning will be detected using relevant ICD10-AM codes. The VEMD injury data are coded according to the National Minimum Data Standards for Injury Surveillance with diagnoses coded according to the ICD-10-AM. ${ }^{16}$ As most of these cases do not contain opioid-specific information, it is anticipated they will be included in the 'all

Table 1 Summary of the search criteria

\begin{tabular}{|c|c|}
\hline Free-text search & $\begin{array}{l}\text { ICD10-AM code (where drug name does not appear in } \\
\text { the text field) }\end{array}$ \\
\hline $\begin{array}{l}\text { [All pharmaceutical opioid drug name including variations } \\
\text { (eg, "Tramadol/ tramal/ zydol", "Morphine/ MS contin/ } \\
\text { MS mono/ kapanol/ anamorph/ sevredol", "Oxycodone/ } \\
\text { oxycodeine/ oxy/ oxycontin/ endone/ targin/ oxynorm/ } \\
\text { proladone/ novacodone")] } \\
\text { AND } \\
\text { [Overdose/poisoning terms included in the text field (eg, } \\
\text { 'overdose' 'od' 'poisoning' 'drug abuse' 'toxicity' 'poison' } \\
\text { 'self harm' 'suicide') } \\
\text { OR } \\
\text { ICD10-AM codes for poisoning by narcotics and } \\
\text { psychodysleptics' appear in one of the three VEMD } \\
\text { diagnosis codes T40.2, T40.3, T40.4] }\end{array}$ & $\begin{array}{l}\text { The following T40 'poisoning by narcotics and } \\
\text { psychodysleptics' codes appear anywhere within the } \\
\text { three VEMD diagnosis codes: } \\
\text { T40.2-other opioids, for example, codeine or } \\
\text { morphine, also hydromorphone, oxycodone, opioid } \\
\text { not elsewhere classified (NEC), hydrocodone } \\
\text { T40.3-methadone } \\
\text { T40.4-other synthetic narcotics, including } \\
\text { pethidine, Opiate NEC, buprenorphine, } \\
\text { dextropropoxyphene, fentanyl, tramadol }\end{array}$ \\
\hline
\end{tabular}

ICD10-AM, International Classification of Diseases 10th Revision, Australian modification; VEMD, Victorian Emergency Minimum Dataset. 
pharmaceutical opioid' overdoses category, but not included in the opioid-specific analyses.

The following ICD10-AM T40 'poisoning by narcotics and psychodysleptics' codes will be searched for anywhere within the three VEMD diagnosis codes:

- T40.2-other opioids, for example, codeine or morphine, also hydromorphone, oxycodone, opioid not elsewhere classified (NEC), hydrocodone.

- T40.3-methadone.

- T40.4other synthetic narcotics, including pethidine, opiate NEC, buprenorphine, dextropropoxyphene, fentanyl, tramadol.

Cases will be selected for all intent groups (unintentional, intentional self-harm, assault, maltreatment and neglect, other and undertermined intent). Consistent with previous overdose research, cases will be restricted to those aged 12 years and over to omit cases of accidental poisoning by children. ${ }^{18} 19$ This age limit means that it is likely most individuals who used the pharmaceutical opioid were aware it was a drug.

\section{Pharmaceutical opioid sales data}

The utilisation of individual opioids will be estimated through the sale of each opioid to Victorian community pharmacies. This sales data (unit sales by strength of product for each of the opioids involved) will be accessed via a third party access agreement with the multinational health information and clinical research company IQVIA (iqvia.com). Sales data will represent the entire Victorian population (ie, population level data) and do not contain individual identifiers.

The total amount of each opioid supplied per month will be calculated in milligrams, and converted into oral morphine equivalents (OME) so the analgesic effect of different opioids can be represented on the same scale. ${ }^{20}$ We will then use these OME to calculate a supply-adjusted rate of ED presentations, a method consistent with previous studies of pharmaceutical opioid related harm. ${ }^{45}$

\section{Statistical analysis}

Descriptive statistics will be used to summarise prescription opioid-related ED presentations (eg, annual supplyadjusted rates for each opioid in Victoria, for each year in the study period). Units will be presentations per 100 $000 \mathrm{mg} \mathrm{OME} .^{20} \mathrm{CIs}$ for rates will be calculated using the exact method based on the Poisson distribution. Statistics based on numbers less than 5 will not be reported; however, zeroes are preserved. Data may be aggregated (eg, reported quarterly rather than by month) to ensure cell sizes of at least 5 .

\section{Aim 1 analysis plan—supply-adjusted rates of harm}

Poisson regression will be used to generate incident rate ratios with $95 \%$ CIs for overall supply adjusted rates, allowing for simultaneous estimation of individual opioid effect sizes and their change over time. This will permit statistical comparisons of differences in supply-adjusted rates across different opioids. Averaged monthly rates of presentations by year examined for each pharmaceutical opioid will be reported. Rates of presentations will be calculated based on either monthly or 3-month intervals (or longer time intervals if required, to enable minimum cell sizes of 5), adjusted for supply of that opioid using Victorian pharmaceutical sales data. Where cell sizes are $<5$, to preserve anonymity, we will report the average of all cells with 1-4 cases, rather than the actual number of cases in that cell.

\section{Aim 2 analysis plan —characteristics of ED presentations}

We will use multinomial logistic regression to analyse opioid-poisoning characteristics. Characteristics include the patient demographics of age, sex, region, country of birth and socioeconomic status; and the presentation characteristics of whether the overdose was intentional, admission outcome and clinical severity (table 2). Separate regressions will be run with each attendance characteristic serving as the primary independent variable. Opioid type will be the outcome variable in all regressions with morphine, a mid-potency opioid commonly used as a standard reference for calculating opioid doses, ${ }^{21}$ as the reference category. Results will be expressed as the estimated odds of each opioid (relative to morphine) being involved in the ED presentation for a particular attendance characteristic. In addition, year will be included as an independent variable in all regressions to assess whether characteristics changed over time- the potential of time as an effect modifier in the relationship between the attendance characteristic and opioid type will be evaluated by testing the statistical interaction between the two independent variables in the regression model. When considering the triage severity, the model will be also be adjusted for by age and sex, in addition to other characteristics identified in univariate analyses to be associated with severity of overdose. VEMD categories will be aggregated where necessary to ensure that all analyses report cell sizes of at least five.

All quantitative analyses will be conducted in SAS or Stata, with $p$ values less than 0.05 considered significant.

\section{Handling known or expected missing data}

To minimise missing data to enable us to preserve reportable cells for aim 1 (ie, no cell less than 5), we will aggregate the data into multiple month blocks (eg, 3-monthly periods) as we anticipate some opioids will have low counts in individual months and these data would not be able to be reported.

In calculating rates of presentations, if the numerator is less than 10 for the analysis period, we will aggregate the available data over multiple periods, for example, to provide an estimate of the rate for the past year rather than a 3-month block, using standard procedures developed by the data custodians to ensure confidentiality with reported data.

Deaths in hospital would be recorded in the VEMD. It is of note, however, that these are very rare. Deaths on arrival at ED are even rarer -5 or less have been recorded 
Table 2 Variables and response options to be examined in association with pharmaceutical opioid-related emergency department presentations, by opioid-type

\begin{tabular}{|c|c|c|}
\hline Category & Variable & Variable options \\
\hline \multirow[t]{5}{*}{ Patient demographics } & Age & $\geq 12$ years; extracted in 5 -year blocks \\
\hline & Sex & $\begin{array}{l}\text { Males, females, total (including other genders with cell sizes too small to be } \\
\text { extracted separately) }\end{array}$ \\
\hline & Region & Metro, regional/rural, interstate/overseas, unknown \\
\hline & Country of birth & $\begin{array}{l}\text { Australia, overseas (presented as an English speaking country, or non-English } \\
\text { speaking country if cell sizes allow). The } 12-\text { month substance use disorder } \\
\text { prevalence is } 6 \% \text { for Australian born residents, } 4.4 \% \text { if the birthplace was } \\
\text { another English-speaking country, and } 1.6 \% \text { for a non-English speaking } \\
\text { country. }\end{array}$ \\
\hline & Patient SEIFA & $\begin{array}{l}\text { Socio-Economic Indexes for Areas (SEIFA) is a proxy measure for } \\
\text { socioeconomic status based on home postcode. }{ }^{28} \text { Australians living in the } \\
\text { lowest socioeconomic areas have the highest burden of disease and highest } \\
\text { rates of opioid prescriptions. }{ }^{2}\end{array}$ \\
\hline $\begin{array}{l}\text { Admission outcome } \\
\text { (proxy of severity) }\end{array}$ & & $\begin{array}{l}\text { i. Discharge to home/ returning to usual residence ii. Admission to ward/ } \\
\text { procedure room - this campus (note, this is also a proxy for clinical severity) iii. } \\
\text { Transfer to another hospital campus iv. Departure before treatment completed }\end{array}$ \\
\hline $\begin{array}{l}\text { Triage severity of } \\
\text { presentation (proxy of } \\
\text { severity) }\end{array}$ & $\begin{array}{l}\text { Australasian } \\
\text { Triage Scale (ATS) } \\
\text { Category }\end{array}$ & $\begin{array}{l}\text { Each of the five categories relates to treatment acuity and the maximum waiting } \\
\text { time for medical assessment and treatment. } \\
\text { i. ATS } 1 \text { (Immediate) ii. ATS } 2 \text { (10 min) iii. ATS } 3 \text { ( } 30 \text { min) iv. ATS } 4 \text { (60 min) } \\
\text { v. ATS } 5 \text { (120 min) }\end{array}$ \\
\hline
\end{tabular}

for all causes in the VEMD database in the past 10 years. As such, we will not include deaths in our primary analyses due to limitations on reporting small cell sizes.

\section{Ethics}

Ethics approval was obtained from the Monash University Human Research Ethics Committee for VISU to analyse VEMD data for injury surveillance purposes (21427).

This approval requires strict conditions for the storage, retention and use of the VEMD data to protect privacy and confidentiality and all unit level data are stored and analysed onsite at VISU. Due to the sensitivity and potentially identifiable nature of the data, line item data cannot be provided to researchers outside the VISU without additional ethical approval. As per standard procedures with the data custodians, cells of $<5$ will not be reported, though zeroes will be preserved. The sales data are held by IQVIA and ethics approval is not required for the use and publication of sales data.

\section{Data statement}

Researchers interested in using these data may approach VISU and IQVIA. Access fees for data and/or analyses may apply.

\section{Dissemination}

We will present project findings in a peer-reviewed journal article as well as at relevant scientific conferences. Findings will be reported in accordance with the REporting of studies Conducted using Observational
Routinely-collected health Data statement, an extension of the Strengthening the Reporting of Observational Studiesin Epidemiology statement for reporting items specific to observational studies using routinely collected health data. ${ }^{22}$

\section{Patient and public involvement}

Patients and the public were not involved in the design of the study.

\section{DISCUSSION}

This study aims to compare rates and characteristics of harm related to specific pharmaceutical opioids. Emergency department presentations for opioid poisoning will be compared across nine of the most commonly used pharmaceutical opioids in Australia.

\section{Strengths}

These findings will provide a detailed understanding of the relative contribution of well-established and newer pharmaceutical opioids to poisonings in Victoria, which to date have only been examined as a category of "pharmaceutical opioids', or by the three ICD-10 categories methadone, 'other opioids' (eg, codeine or morphine, oxycodone) and other synthetic narcotics (eg, tramadol, fentanyl). These coding typically do not enable disaggregation of opioids with very different profiles (such as tramadol and fentanyl) nor have they been able to 
examine newer opioids with limited postmarketing surveillance such as tapentadol, which have seen large increases in prescribing in recent years. ${ }^{6} 23$

ED presentations are used to provide a robust (but conservative) overview of opioid poisoning in Victoria. The dataset represents the population of Victoria (as represented by the hospitals that contribute to the VEMD) and will give the most complete picture of types of pharmaceutical opioid poisoning in ED settings in Victoria to date.

ED data are a population-level measure that reflects real world harms with a broader capture than abuse liability studies, clinical trials or spontaneous adverse event reporting systems.

Opioid poisoning is a key measure of opioid-related harm that is driving policy change in Australia. These data are carefully hand coded and can inform both the relative frequency and severity of presentations associated with different pharmaceutical opioids. Importantly, this study will extract data using free-text searches as well as ICD-codes, the latter of which has been determined to substantially underestimate drug related harms in Victorian EDs. ${ }^{1024}$ Furthermore, every case will be manually checked to confirm it represents a pharmaceutical opioid poisoning.

Sales data are more inclusive than prescription data as it includes private prescription items and items that can be sold over the counter. Up to a fifth of opioids are not accounted for by government subsidised prescriptions, ${ }^{25}$ and this disproportionately affects some opioids such as codeine and tapentadol. So, the use of sales data will more closely approximate supply and actual use compared with the use of prescription subsidy data.

This study will complement other research conducted comparing harms across pharmaceutical opioids. We will specifically examine ED opioid poisoning in this study, while previous research has examined a range of ambulance-attended opioid-related harms. ${ }^{6}$ Both supplyadjusted rates of harm will be available for triangulation for a similar time period within the state of Victoria providing a more comprehensive understanding of harms.

\section{Limitations}

Reliable details on the source of opioid or nature of use are not routinely recorded in the VEMD, so details such as whether harms are related to non-medical use versus therapeutic use will not be able to be explored. Similarly, the source of the drug is not typically recorded by the ED clinician as it is not a required clinical field in the database. As these are not consistently coded or reliable variables, we do not intend to use them in this analysis. As such, these findings will primarily provide information on rates and characteristics of opioid poisoning presentations rather than the context of use.

The rates calculated using VEMD data are expected to under-represent total pharmaceutical opioid poisonings. First, around one-third of ED opioid cases do not have a specific opioid documented ${ }^{26}$-for example, due to lack of detailed ICD10-AM codes for each drug type, or as the coder is unable to ascertain the type of opioid involved. This may lead to an underestimation of the rates of harm for each opioid, though we do not expect this to introduce bias to specific opioids. Second, the data will not capture overdoses managed outside the ED, for example, by paramedics and patient was not transferred on to the $\mathrm{ED}$, noting that these are captured elsewhere, ${ }^{6}$ or not captured by a medical professional.

Finally, there may be unmeasured confounders such as the likelihood of an individual being prescribed a specific opioid that we cannot account for using a naturalistic study design. It is of note, however, patients who use opioids extramedically are usually excluded from the trials which are able to randomise potential confounders.

While the analysis will under-represent total opioidrelated harm in the general population, it will give the most complete picture of pharmaceutical opioid poisoning in ED settings.

\section{Author affiliations}

${ }^{1}$ Monash Addiction Research Centre, Eastern Health Clinical School, Monash University, Frankston, Victoria, Australia

${ }^{2}$ Victorian Injury Surveillance Unit, Monash University Accident Research Centre, Monash University, Clayton, Victoria, Australia

${ }^{3}$ Turning Point, Eastern Health Clinical School, Monash University, Richmond, Victoria, Australia

\section{Twitter Suzanne Nielsen @drsuzinielsen}

Contributors SN conceptualised the study with input into the study design from TL, PS, JH and JB-G. TL wrote the initial draft, which was revised with input from in collaboration with all authors. PS developed the analysis plan with input from all authors. All authors have read and approved the revised protocol manuscript.

Funding The study is funded by an untied educational grant from Seqirus. SN is the recipient of an NHMRC Career Development Fellowship (\#1163961). Prior to publication, Seqirus will have the opportunity to review the manuscript and provide comment on factual inaccuracies, if identified.

Disclaimer The funders will have no role in the study design, study conduct, analysis or data interpretation.

Competing interests In the past 5 years, SN has been an investigator on untied education grants from Indivior, unrelated to the current work. SN has provided training to health care professionals on identifying and treating codeine dependence for which her institution has received payment from Indivior. DIL has received speaking honoraria from the following: Astra Zeneca, Indivior, JanssenCilag, Lundbeck, Servier and Shire, and has participated on Advisory Boards for Indivior and Lundbeck.

Patient and public involvement Patients and/or the public were not involved in the design, or conduct, or reporting, or dissemination plans of this research.

Patient consent for publication Not required.

Provenance and peer review Not commissioned; externally peer reviewed.

Open access This is an open access article distributed in accordance with the Creative Commons Attribution Non Commercial (CC BY-NC 4.0) license, which permits others to distribute, remix, adapt, build upon this work noncommercially, and license their derivative works on different terms, provided the original work is properly cited, appropriate credit is given, any changes made indicated, and the use is non-commercial. See: http://creativecommons.org/ licenses/by-nc/4.0/.

ORCID iD

Suzanne Nielsen http://orcid.org/0000-0001-5341-1055 


\section{REFERENCES}

1 Seth P, Scholl L, Rudd RA, et al. Overdose Deaths Involving Opioids, Cocaine, and Psychostimulants - United States, 2015-2016. MMWR Morb Mortal Wkly Rep 2018;67:349-58.

2 Australian Institute for Health and Welfare. Opioid harm in Australia and comparisons between Australia and Canada; 2018.

3 Vivolo-Kantor AM, Seth P, Gladden RM, et al. Vital Signs : Trends in Emergency Department Visits for Suspected Opioid Overdoses United States, July 2016-September 2017. MMWR Morb Mortal Wkly Rep 2018;67:279-85.

4 Roxburgh A, Hall WD, Dobbins T, et al. Trends in heroin and pharmaceutical opioid overdose deaths in Australia. Drug Alcohol Depend 2017;179:291-8.

5 Murphy DL, Lebin JA, Severtson SG, et al. Comparative rates of mortality and serious adverse effects among commonly prescribed opioid analgesics. Drug Saf 2018;41:787-95.

6 Nielsen S, Crossin R, Middleton M, et al. Comparing rates and characteristics of ambulance attendances related to extramedical use of pharmaceutical opioids in Victoria, Australia from 2013 to 2018. Addiction 2020;115:1075-87.

7 World Health Organization. Community management of opioid overdose. Geneva, Switzerland: WHO Document Production Services, 2014

8 Caudarella A, Dong H, Milloy MJ, et al. Non-Fatal overdose as a risk factor for subsequent fatal overdose among people who inject drugs. Drug Alcohol Depend 2016;162:51-5.

9 Stoové MA, Dietze PM, Jolley D. Overdose deaths following previous non-fatal heroin overdose: record linkage of ambulance attendance and death registry data. Drug Alcohol Rev 2009;28:347-52.

10 Lam T, Kuhn L, Hayman J, et al. Recent trends in heroin and pharmaceutical opioid-related harms in Victoria, Australia up to 2018. Addiction 2020;115:261-9.

11 PLOS Medicine Editors. Observational studies: getting clear about transparency. PLoS Med 2014;11:e1001711-e.

12 West R. Trial protocols. Addiction 2012;107:1544.

13 Australian Bureau of Statistics. 3101.0 - Australian Demographic Statistics, 2018. Available: http://www.abs.gov.au/ausstats/abs@.nsf/ $\mathrm{mf} / 3101.0$

14 Pennington Institute. Australia's Annual Overdose Report 2019. Melbourne Penington Institute; 2019.

15 Australian Institute for Health and Welfare. Australia's health 2018 Canberra: Australian Institute for Health and Welfare; 2018.
16 Department of Health and Human Services. Victorian emergency minimum dataset (VEMD). Australia State Government of Victoria; 2019. https://www2.health.vic.gov.au/hospitals-and-health-services/ data-reporting/health-data-standards-systems/data-collections/ vemd

17 Nielsen S, Crossin R, Middleton M, et al. Comparing rates and characteristics of ambulance attendances related to extramedical use of pharmaceutical opioids in Australia: a protocol for a retrospective observational study. BMJ Open 2019;9:e029170.

18 Rhodes AE, Bethell J, Spence J, et al. Age-sex differences in medicinal self-poisonings: a population-based study of deliberate intent and medical severity. Soc Psychiatry Psychiatr Epidemiol 2008;43:642-52.

19 Gunnell D, Ho D, Murray V. Medical management of deliberate drug overdose: a neglected area for suicide prevention? Emerg Med $\mathrm{J}$ 2004;21:35-8.

20 Nielsen S, Degenhardt L, Hoban B, et al. A synthesis of oral morphine equivalents (OME) for opioid utilisation studies. Pharmacoepidemiol Drug Saf 2016;25:733-7.

21 Nielsen S, Degenhardt L, Hoban B, et al. A synthesis of oral morphine equivalents (OME) for opioid utilisation studies. Pharmacoepidemiol Drug Saf 2016;25:733-7.

22 Benchimol El, Smeeth L, Guttmann A, et al. The reporting of studies conducted using observational Routinely-collected health data (record) statement. PLoS Med 2015;12:e1001885-e.

23 Peacock A, Gisev N, Memedovic S, et al. Opioid use and harms associated with a sustained-release tapentadol formulation: a post-marketing surveillance study. Drug Alcohol Depend 2020;206:107697.

24 Di Rico R, Nambiar D, Stoové M, et al. Drug overdose in the ED: a record linkage study examining emergency department ICD-10 coding practices in a cohort of people who inject drugs. BMC Health Serv Res 2018;18:945.

25 Gisev N, Pearson S-A, Karanges EA, et al. To what extent do data from pharmaceutical claims under-estimate opioid analgesic utilisation in Australia? Pharmacoepidemiol Drug Saf 2018;27:550-5.

26 Hayman J, Berecki-Gisolf J, Nielsen S, et al. Trends in pharmaceutical Opioid-related harm in Victoria, 2008/09 to 2017/18. Melbourne, Australia: Victorian Injury Surveillance Unit, 2019.

27 Slade T, Johnston A, Teesson M, et al. The mental health of Australians 2. Report on the 2007 national survey of mental health and wellbeing. Canberra: Department of Health and Ageing, 2009.

28 Australian Bureau of Statistics. Measures of socioeconomic status; 2011. 